\title{
Prática clínica do Enfermeiro na atenção à saúde do idoso portador de covid-19
}

\author{
Clinical practice of Nurses in health care for elderly patients with covid-19 \\ Práctica clínica de Enfermeras en la atención de la salud de pacientes ancianos con covid-19
}

Recebido: 04/06/2021 | Revisado: 10/06/2021 | Aceito: 21/06/2021 | Publicado: 04/07/2021

Victor Hugo de Paula Flauzino

ORCID: https://orcid.org/0000-0001-5156-0030

Associação Brasileira de Enfermeiros Cientistas, Brasil

E-mail: prof.victorflauzino@unyleya.edu.br

Judith Victoria Castillo Mejía

ORCID: https://orcid.org/0000-0001-5257-6878

Associação Brasileira de Enfermeiros Cientistas, Brasil

E-mail: iker07castillo@live.com

Priscila Gramata da Silva Vitorino

ORCID: https://orcid.org/0000-0002-1201-6945

Associação Brasileira de Enfermeiros Cientistas, Brasil

E-mail: Prigramaenf@yahoo.com.br

Thays Vieira Cusato

ORCID: https://orcid.org/0000-0002-3766-6530

Associação Brasileira de Enfermeiros Cientistas, Brasil

E-mail: thays.vieira.cusato@gmail.com

Daiana Moreira Gomes

ORCID: https://orcid.org/0000-0001-9387-0619

Associação Brasileira de Enfermeiros Cientistas, Brasil

E-mail: daigomes_87@hotmail.com

Daniele Vignoli Ribeiro

ORCID: https://orcid.org/0000-0001-5296-8302

Associação Brasileira de Enfermeiros Cientistas, Brasil

E-mail: vignoliribeiro@gmail.com

Luana de Oliveira Hernandes

ORCID: https://orcid.org/0000-0001-8828-7146

Associação Brasileira de Enfermeiros Cientistas, Brasil

E-mail: luanaoliveira2306@gmail.com

Thais Oliveira de Paula Lima

ORCID: https://orcid.org/0000-0002-0706-1098

Associação Brasileira de Enfermeiros Cientistas, Brasil

E-mail: thaisoplima@gmail.com

Natasha Vila Chã

ORCID: https://orcid.org/0000-0002-9781-454X Associação Brasileira de Enfermeiros Cientistas, Brasil

E-mail: natashavilacha@hotmail.com

Jonas Magno dos Santos Cesário

ORCID: https://orcid.org/0000-0003-1785-3555

Associação Brasileira de Enfermeiros Cientistas, Brasil

E-mail: prof.jonasmagno@unyleya.edu.br

\begin{abstract}
Resumo
Assim como no restante do mundo, o Brasil enfrentou um rápido avanço da contaminação por covid-19 e em junho de 2021, mais de 16 milhões de brasileiros já haviam se contaminado e os óbitos eram de aproximadamente 500 mil. Entre os grupos mais afetados, estavam pessoas com mais de 60 anos de idade, principalmente aquelas que possuíam algum tipo comorbidade. Devido a rápida evolução e piora dos pacientes nesta faixa etária, o enfermeiro deve adotar condutas que contribuam para a melhora deste paciente, assim como medidas que evitem a propagação do vírus. Nesse contexto, o objetivo deste estudo foi descrever prática clínica do enfermeiro na atenção à saúde do idoso portador de covid-19. A metodologia foi um revisão bibliográfica de abordagem descritiva e qualitativa de estudos públicados nos anos de 2020 e 2021. Diante da complexidade do processo de envelhecimento humano, com peculiaridades próprias, aliada à alta incidência das doenças crônicas e suas repercussões no corpo humano, evidencia-se a necessidade de atenção específica aos idosos, o que inclui ações de prevenção, tratamento e reabilitação. Em relação ao idoso portador do covid-19, cabe ressaltar a importância de um atendimento rápido e eficiente, que garanta o distanciamento do idoso acometido pela doença das demais pessoas, mas que ainda sim seja um cuidado humanizado. Novos estudos científicos são necessários para aperfeiçoar a prática clínica do enfermeiro no combate ao novo coronavírus em idosos, com foco na melhora da qualidade de vida e reabilitação para aqueles recuperados.
\end{abstract}


Palavras-chave: Idoso; Infecções por coronavírus; Cuidados de enfermagem.

\begin{abstract}
As in the rest of the world, Brazil faced a rapid advance of contamination by covid-19 and in June 2021, more than 16 million Brazilians had already been infected and deaths were approximately 500 thousand. Among the most affected groups were people over 60 years of age, especially those who had some type of comorbidity. Due to the rapid evolution and worsening of patients in this age group, nurses must adopt measures that contribute to the improvement of this patient, as well as measures to prevent the spread of the virus. In this context, the aim of this study was to describe the clinical practice of nurses in the health care of elderly people with covid-19. The methodology was a bibliographic review with a descriptive and qualitative approach of studies published in 2020 and 2021. Given the complexity of the human aging process, with its own peculiarities, combined with the high incidence of chronic diseases and their repercussions on the human body, and the need for specific attention to the elderly, which includes prevention, treatment, and rehabilitation actions. Regarding the elderly with covid-19, it is worth emphasizing the importance of fast and efficient care, which guarantees the distance between the elderly affected by the disease from other people, but still providing humanized care. New scientific studies are needed to improve the clinical practice of nurses in combating the new coronavirus in the elderly, with a focus on improving the quality of life and rehabilitation for those recovered.
\end{abstract}

Keywords: Elderly; Coronavírus infections; Nursing care.

\title{
Resumen
}

Como en el resto del mundo, Brasil enfrentó un rápido avance de contaminación por covid-19 y en junio de 2021, más de 16 millones de brasileños ya habían sido infectados y las muertes fueron aproximadamente 500 mil. Entre los grupos más afectados se encontraban las personas mayores de 60 años, especialmente aquellas que presentaban algún tipo de comorbilidad. Debido a la rápida evolución y agravamiento de los pacientes en este grupo de edad, las enfermeras deben adoptar medidas que contribuyan a la mejoría de este paciente, así como medidas para prevenir la propagación del virus. En este contexto, el objetivo de este estudio fue describir la práctica clínica del enfermero en el cuidado de la salud de personas mayores con covid-19. La metodología fue una revisión bibliográfica con enfoque descriptivo y cualitativo de estudios publicados en 2020 y 2021. Dada la complejidad del proceso de envejecimiento humano, con sus peculiaridades, combinado con la alta incidencia de enfermedades crónicas y sus repercusiones en el cuerpo humano, y la necesidad de una atención específica a las personas mayores, que incluye acciones de prevención, tratamiento y rehabilitación. En cuanto a las personas mayores con covid-19, cabe destacar la importancia de una atención rápida y eficaz, que garantice la distancia entre las personas mayores afectadas por la enfermedad y las demás personas, pero aun brindando una atención humanizada. Se necesitan nuevos estudios científicos para mejorar la práctica clínica de las enfermeras en la lucha contra el nuevo coronavirus en los ancianos, con un enfoque en la mejora de la calidad de vida y rehabilitación de los recuperados.

Palabras clave: Ancianos; Infecciones por coronavírus; Cuidados de enfermería.

\section{Introdução}

O atual cenário causado pelo Coronavírus-2 (Sars-Cov-2) começou no final de 2019, com a notificação de um caso de uma gripe misteriosa, na cidade de Wuhan, China. A pandemia mundial causada pelo covid-19 foi declarada em 11 de março de 2020. Desde então, inúmeras mudanças foram necessárias nos sistemas de saúde do mundo todo, pois passaram a atender uma grande demanda de pacientes infectados, o exigiu uma adaptação rápida dos profissionais de enfermagem, tanto para atuar na recuperação dos pacientes, quanto para controlar a disseminação do vírus (Souza et al., 2021).

Em 2020 o número de infectados mundialmente pelo covid-19 cresceu muito e em junho já eram 6 milhões de casos, em dezembro esse número ultrapassou os 70 milhões. Um ano depois, em junho de 2021, este número ultrapassou a marca dos 170 milhões de indivíduos infectados. Nessa mesma o Brasil enfrentava um rápido avanço da doença, com mais de 16 milhões de casos e aproximadamente 500 mil óbitos. As pessoas com mais de 60 anos de idade e que possuíam algum tipo de comorbidade representaram a faixa etária mais atingida desde o início da pandemia, no entanto com o avanço da contaminação entre os jovens, foi possível observar um aumento significativo de casos e óbitos entre este último grupo (John Hopkins Coronavirus Resource Center, 2021).

O covid-19 desencadeia a síndrome respiratória aguda grave, o que preocupa a equipe de saúde devido a rápida piora do paciente. Ao considerar sua forma acelerada de transmissão e a ausência de medidas farmacológicas efetivas, é possível 
afirmar que até o presente momento as principais estratégias e ações para desacelerar a disseminação do coronavírus são: a higienização das mãos, uso de álcool em gel, utilização de máscara, distanciamento e isolamento social (Lipsitch, Swerdlow e Finelli, 2020 \& Souza et al., 2020).

Estudos comprovam que os números de casos de contaminação e morbimortalidade, especialmente nas pessoas idosas, são elevados. Durante a pandemia, a contaminação da população idosa pelo covid-19 foi uma das principais preocupações dos profissionais de saúde, que precisou adaptar suas rotinas e a forma de atendimento, para proteger essa população mais vulnerável. Como nessa faixa etária existe uma prevalência maior de doenças crônicas, sem uma rápida resposta dos profissionais que cuidam destes pacientes, a doença se propagaria de forma mais rápida (Lloyd-Sherlock et al., 2020 \& Santos et al., 2020).

Nesse contexto a equipe de enfermagem precisou se adequar para atender pacientes na urgência e emergência e seguir as estratégias que impedissem a disseminação da doença, assim como, identificar os principais problemas causados pela doença, a ponto de intervir de forma eficaz para diminuir os riscos de contaminação e os riscos de infecção, além de, promover a saúde do paciente. Diante desse contexto, surgiu a seguinte indagação: como é prática clínica do enfermeiro na atenção à saúde do idoso portador de covid-19?

Ao considerar a fragilidade dos idosos acometido pelo vírus da covid-19, o enfermeiro se viu diante de uma situação complexa, que exige atendimento que garanta a integridade destes pacientes e que contribua para não disseminar o vírus. Com isto o presente estudo possui o objetivo de descrever a prática clínica do enfermeiro na atenção à saúde do idoso portador de covid-19.

\section{Metodologia}

O estudo foi realizado por meio de uma revisão bibliográfica de abordagem descritiva e qualitativa, que é descrita por Cesário, Flauzino e Mejia (2020) como um tipo de pesquisa fundamentada em materiais que já foram construídos, o que incluí artigos científicos publicados em periódicos acadêmicos.

$\mathrm{Na}$ primeira etapa, buscou-se reunir evidências para responder a seguinte indagação: como é prática clínica do enfermeiro na atenção à saúde do idoso portador de covid-19? No DeCS (Descritores em Ciências da Saúde), encontrou-se os seguintes descritores: idoso, infecções por coronavírus e cuidados de enfermagem. Os bancos de dados utilizados foram o Google Acadêmico, BVS (biblioteca virtual em saúde) e SciELO (Scientific Electronic Library Online). No Google Acadêmico utilizou-se cada um dos descritores entre aspas ("“). Na BVS foi utilizado a opção pesquisa avançada, selecionada as bases da BDENF (Banco de Dados em Enfermagem), LILACS (Literatura Latino-Americana e do Caribe em ciências da saúde) e o operador lógico booleano "OR" e "AND". Na SciELO, foi utilizada a opção pesquisa avançada e o operador lógico booleano "OR" e "AND".

Estabeleceu-se como critérios de inclusão os artigos acadêmicos publicados entre 2020 e 2021, na língua portuguesa, disponíveis de forma gratuita e nos bancos de dados já mencionados, que respondessem à pergunta de pesquisa. Excluíram-se artigos repetidos encontrados nas bases de dados, resumos, artigos inferiores a 2020 e artigos que não respondiam o problema da pesquisa. A coleta dos dados foi realizada no mês de março, por 3 pesquisadores de forma independente. Os resultados das buscas pelos dados e do número final de publicações nessa revisão foram apresentados conforme orientações do JBI e prisma na forma de fluxograma, conforme a Figura 1 (Peters, 2015). 
Figura 1. Diagrama de fluxo dos artigos encontrados.

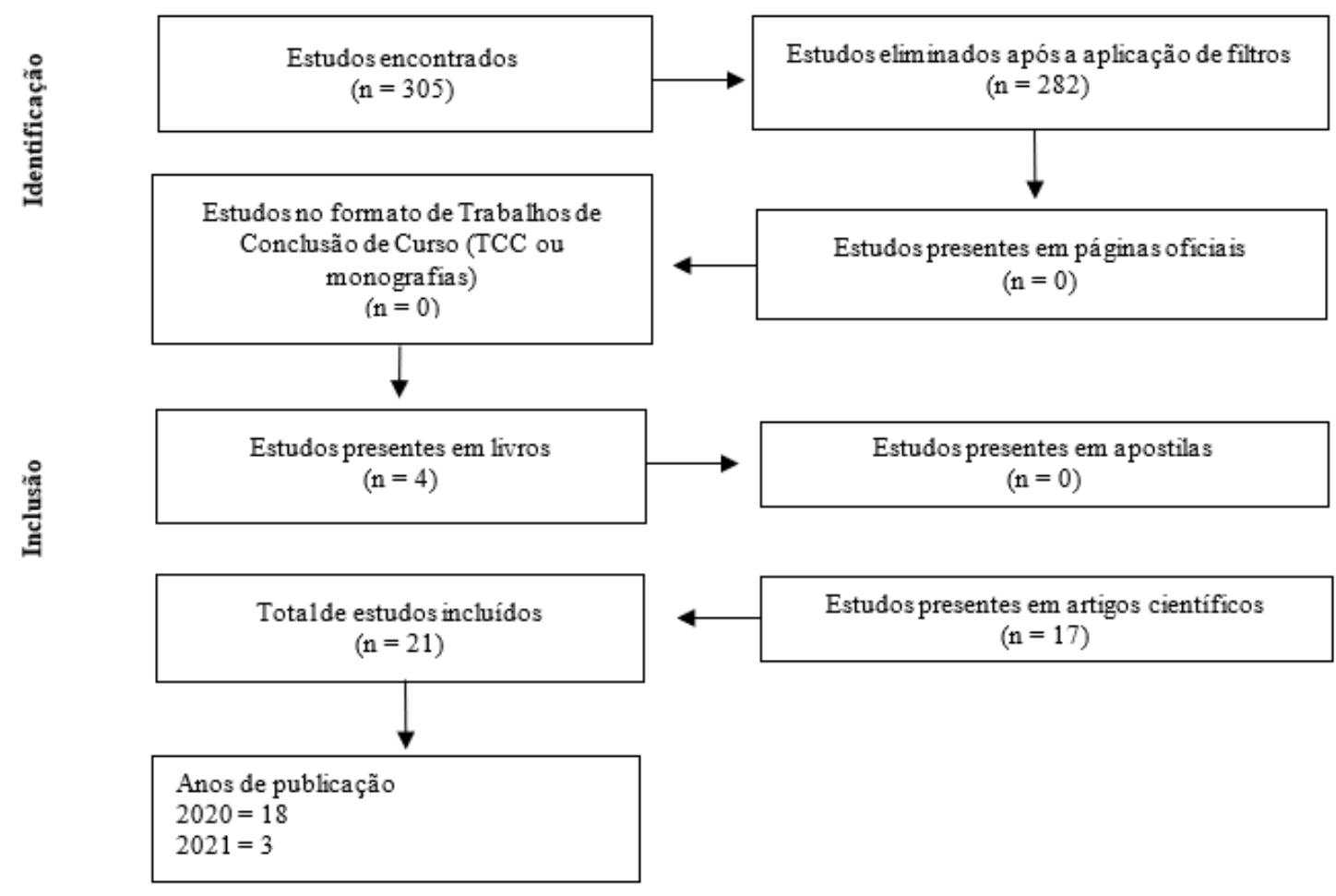

Fonte: Autores (2021).

\section{Resultados}

A Tabela 1, representada abaixo, foi desenvolvida para mostrar a distribuição inicial dos artigos científicos encontrados nas bases de dados da BVS, ScIELO e Google Acadêmico. É possível notar que o maior aproveitamento de periódicos foi na base do Google Acadêmico com 19 artigos incluídos, na BVS foram 5 periódicos incluídos e na ScIELO, foram incluídos somente 7 artigos científicos, o que totalizou uma amostra final de 21 artigos científicos.

Tabela 1 - Resultados da busca nas bases de dados.

\begin{tabular}{|lcc|}
\hline Base de dados & Total & Artigos \\
& Incluídos \\
\hline SciELO & 55 & 7 \\
BVS & 130 & 5 \\
\hline Google acadêmico & 120 & 19 \\
Total & $\mathbf{3 0 5}$ & $\mathbf{2 1}$ \\
\hline
\end{tabular}

Fonte: Autores (2021).

O Quadro 1 foi desenvolvido para apresentar um melhor entendimento dos periódicos selecionados nas bases de dados supracitadas acima. No Quadro 1 é possível notar a relação dos artigos científicos com os objetivos da pesquisa, com isto foi confeccionado com as seguintes variáveis: autor, título, objetivo central e tipo de estudo. Antes de realiza a discussão do trabalho a organização dos estudos no Quadro 1 contribui diretamente para comparar os assuntos abordados durante a pesquisa. 
Quadro 1 - Artigos inclusos neste estudo.

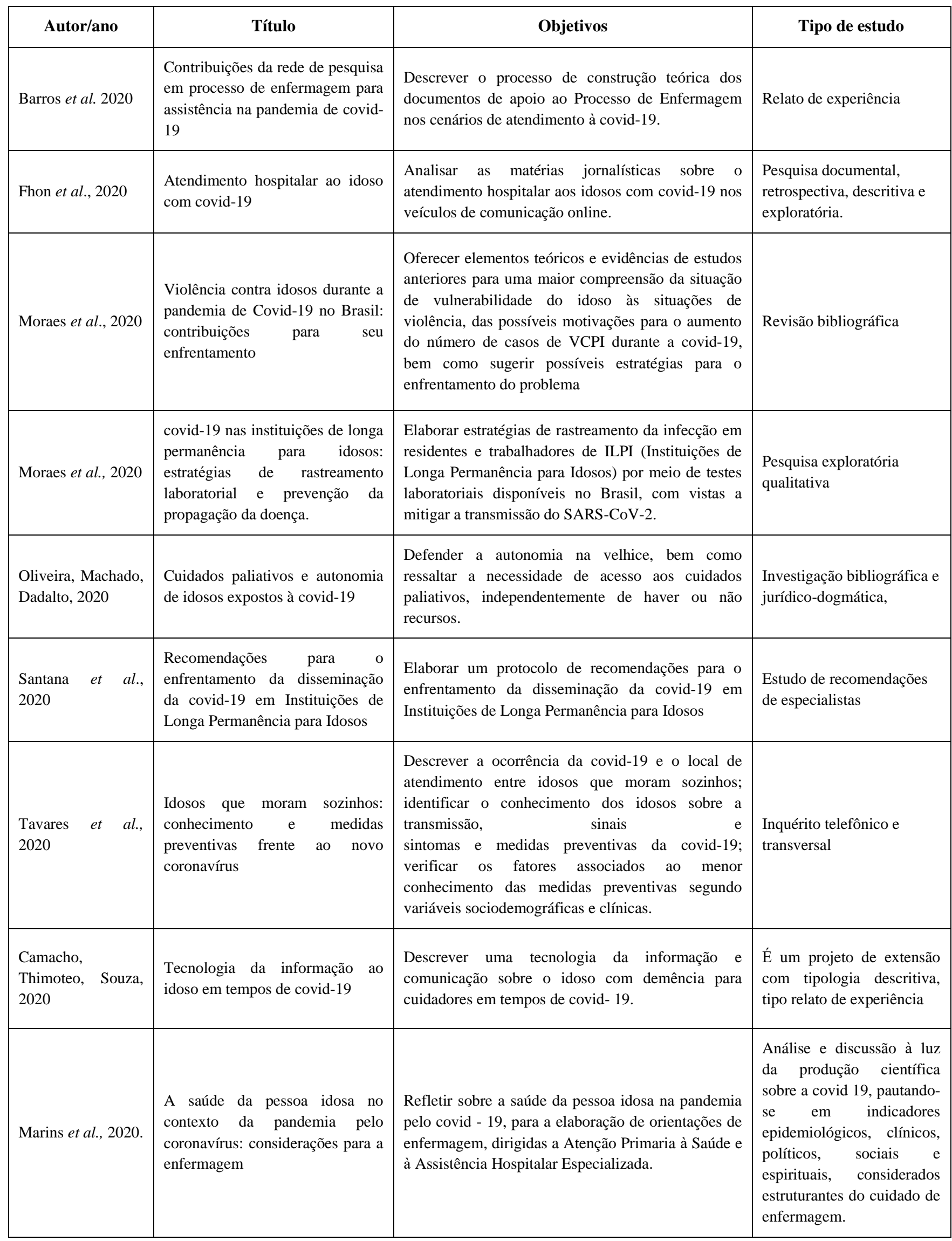




\begin{tabular}{|c|c|c|c|}
\hline $\begin{array}{l}\text { Oliveira; Machado; } \\
\text { Dadalto, } 2020\end{array}$ & $\begin{array}{l}\text { Cuidados paliativos e autonomia } \\
\text { de idosos expostos à covid-19 }\end{array}$ & $\begin{array}{l}\text { Defender a autonomia na velhice, bem como } \\
\text { ressaltar a necessidade de acesso aos cuidados } \\
\text { paliativos, independentemente de haver ou não } \\
\text { recursos }\end{array}$ & $\begin{array}{l}\text { Investigação bibliográfica e } \\
\text { jurídico-dogmática }\end{array}$ \\
\hline Pereira et al., 2020. & $\begin{array}{l}\text { Medidas de enfrentamento para } \\
\text { idosos com covid-19 a luz da } \\
\text { teoria adaptativa de callista roy }\end{array}$ & $\begin{array}{l}\text { Refletir acerca das medidas de enfrentamento para } \\
\text { idosos diagnosticados com covid-19, a luz da teoria } \\
\text { adaptativa de Callista Roy }\end{array}$ & $\begin{array}{l}\text { Estudo descritivo do tipo } \\
\text { teórico-reflexivo }\end{array}$ \\
\hline $\begin{array}{l}\text { Tavares et al., } \\
2020\end{array}$ & 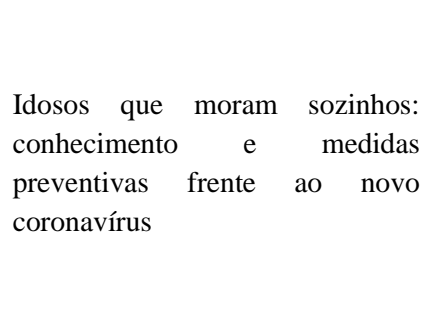 & $\begin{array}{l}\text { Descrever a ocorrência da covid-19 e o local de } \\
\text { atendimento entre idosos que moram sozinhos; } \\
\text { identificar o conhecimento dos idosos sobre a } \\
\text { transmissão, sinais } \\
\text { sintomas e medidas preventivas da covid-19 e } \\
\text { verificar os fatores associados ao menor } \\
\text { conhecimento das medidas preventivas segundo } \\
\text { variáveis sociodemográficas e clínicas. }\end{array}$ & $\begin{array}{l}\text { Inquérito telefônico e } \\
\text { transversal }\end{array}$ \\
\hline $\begin{array}{l}\text { Santana et al., } \\
2020\end{array}$ & $\begin{array}{l}\text { Recomendações para o } \\
\text { enfrentamento da disseminação } \\
\text { da covid-19 em Instituições de } \\
\text { Longa Permanência para Idosos }\end{array}$ & $\begin{array}{l}\text { Elaborar um protocolo de recomendações para o } \\
\text { enfrentamento da disseminação da covid-19 em } \\
\text { Instituições de Longa Permanência para Idosos }\end{array}$ & $\begin{array}{l}\text { Estudo de recomendações } \\
\text { de especialistas }\end{array}$ \\
\hline Fhon et al., 2020 & $\begin{array}{l}\text { Atendimento hospitalar ao idoso } \\
\text { com covid-19 }\end{array}$ & $\begin{array}{l}\text { Analisar as matérias jornalísticas sobre o } \\
\text { atendimento hospitalar aos idosos com covid-19 nos } \\
\text { veículos de comunicação online. }\end{array}$ & $\begin{array}{l}\text { Pesquisa documental, } \\
\text { retrospectiva, descritiva e } \\
\text { exploratória. }\end{array}$ \\
\hline Moraes et al., 2020 & $\begin{array}{l}\text { Violência contra idosos durante a } \\
\text { pandemia de Covid-19 no Brasil: } \\
\text { contribuições para seu } \\
\text { enfrentamento }\end{array}$ & $\begin{array}{l}\text { Oferecer elementos teóricos e evidências de estudos } \\
\text { anteriores para uma maior compreensão da situação } \\
\text { de vulnerabilidade do idoso às situações de } \\
\text { violência, das possíveis motivações para o aumento } \\
\text { do número de casos de VCPI durante a covid-19, } \\
\text { bem como sugerir possíveis estratégias para o } \\
\text { enfrentamento do problema }\end{array}$ & Revisão bibliográfica \\
\hline $\begin{array}{l}\text { Albañil-Delgado, } \\
\text { Machain, } \\
\text { Guerrero- } \\
\text { Castañeda, } 2020\end{array}$ & $\begin{array}{l}\text { Técnicas de arteterapia en casa } \\
\text { como cuidado de Enfermeria para } \\
\text { adultos mayores en contingência } \\
\text { por covid-19 }\end{array}$ & $\begin{array}{l}\text { Apresentar uma breve descrição de técnicas de } \\
\text { terapia com arte no domicílio como cuidados de } \\
\text { enfermagem para idosos em contingência devido ao } \\
\text { covid-19 }\end{array}$ & Descrição de técnica \\
\hline Faria et al., 2020 & $\begin{array}{l}\text { Covid-19: articulação das } \\
\text { políticas de saúde e sociais para } \\
\text { promoção de cuidados seguros } \\
\text { aos idosos }\end{array}$ & $\begin{array}{l}\text { Compreender o processo de articulação das políticas } \\
\text { de saúde e sociais dirigidas aos idosos durante o } \\
\text { estado de emergência por Covid-19 e implicações } \\
\text { para a enfermagem }\end{array}$ & $\begin{array}{l}\text { Estudo qualitativo, } \\
\text { documental e retrospectivo }\end{array}$ \\
\hline $\begin{array}{l}\text { Hammerschimdt \& } \\
\text { Santana. } 2020\end{array}$ & $\begin{array}{l}\text { Saúde do idoso em tempos de } \\
\text { pandemia covid-19 }\end{array}$ & $\begin{array}{l}\text { Abordar de forma reflexiva e crítica aspectos } \\
\text { relacionados à saúde do idoso nos tempos de } \\
\text { pandemia covid-19 }\end{array}$ & Comunicação livre \\
\hline oliveira et al., 2020 & $\begin{array}{l}\text { Principais medidas tomadas para } \\
\text { a mudança dos processos } \\
\text { assistenciais durante a pandemia } \\
\text { por covid-19 }\end{array}$ & $\begin{array}{l}\text { Descrever as principais medidas tomadas para } \\
\text { mudança dos processos assistenciais e de } \\
\text { comunicação interna na vigência da pandemia por } \\
\text { covid-19 de um hospital oncológico }\end{array}$ & $\begin{array}{l}\text { Estudo descritivo de relato } \\
\text { de experiência vivenciada } \\
\text { pelas equipes de Práticas } \\
\text { Assistenciais, Serviço de } \\
\text { Controle de Infecção } \\
\text { Hospitalar, Ensino e } \\
\text { Processos }\end{array}$ \\
\hline $\begin{array}{l}\text { Alves \& Ferreira, } \\
2020\end{array}$ & $\begin{array}{l}\text { Covid-19: reflexão da atuação do } \\
\text { enfermeiro no combate ao } \\
\text { desconhecido }\end{array}$ & $\begin{array}{l}\text { Refletir sobre as consequências da atuação do } \\
\text { enfermeiro perante o surgimento da covid-19 }\end{array}$ & $\begin{array}{l}\text { Artigo } \\
\text { de reflexão }\end{array}$ \\
\hline
\end{tabular}




\section{Discussão}

Os resultados encontrados permitiram dividir e quatro categorias distintas nas quais estão agrupadas nas seguintes formas temáticas: generalidades do covid-19, vulnerabilidade dos idosos ao covid-19 e cuidados de enfermagem ao idoso acometido pelo covid-19. A divisão das temáticas permitiu uma melhor compreensão da prática clínica do enfermeiro, ainda que certos cuidados sejam compartilhados por outros membros da equipe de enfermagem.

\section{Generalidades do covid-19}

Em março de 2020 a Organização Mundial da Saúde (OMS) declarou a infecção por SARS-CoV-2 uma pandemia. O vírus altamente transmissível através do contato direto por disseminação de gotículas respiratórias, na qual os idosos apresentam o grupo com o pior prognóstico para o covid-19. Nesse sentido, faz-se necessário adotar medidas para redução da transmissão, como o distanciamento social e o confinamento dos idosos (Faria et al., 2020; Moraes et al., 2020; Oliveira; Machado; Dadalto, 2020; Santana et al., 2020).

Os sintomas da infecção pelo novo coronavírus preocupam, pois o indivíduo pode apresentar desde um quadro leve até o óbito. Desta maneira, algumas mudanças são necessárias no cuidado em oncologia durante a pandemia de covid-19, quem apresenta o objetivo de promover uma assistência pautada no cuidado seguro e livre de danos que destaca a importância da disponibilização do conhecimento (Oliveira et al., 2020 \& Santana et al., 2020).

O covid-19 causa doença respiratória grave em alguns indivíduos e por apresentar alto índice de transmissibilidade, problema somado a falta de vacina e medicamento específicos, fez com o distanciamento social e as medidas higiênicas com uso de máscaras se tornassem as principais estratégias de combate à pandemia. Identificar os cuidados adequados foram um grande desafio durante a pandemia, no entanto para o grupo formado por idosos com doenças crônicas, o desafio foi ainda maior, pois além da preocupação com a doença, o profissional precisou lidar com a velhice, um processo natural e inevitável, em que deve ser respeitado a vida e aos valores da pessoa idosa. O respeito ao exercício da autonomia destes pacientes, com seu direito de escolha e esclarecimento a respeito do tratamento deve ser observado. O maior número de óbitos também afetou a prática do enfermeiro no que diz respeito aos cuidados paliativos, que passaram a ser mais frequentes. Os cuidados no fim da vida devem respeitar a dignidade, autonomia e o respeito aos valores da pessoa (Oliveira, Machado \& Dadalto, 2020).

\section{Vulnerabilidades dos idosos ao covid-19}

Entende- se que a covid-19 pode trazer sérios riscos à saúde independente de faixa etária da pessoa acometida pela doença. Porém, estudos comprovam que o coronavírus no momento que atinge pessoas com doenças preexistentes, pode trazer um comprometimento ainda maior a saúde. Doenças preexistentes como diabetes, hipertensão, problemas pulmonares, por exemplo, são doenças crônicas que, geralmente, são mais frequentes em pessoas com idade acima de 60 anos. Por apresentarem maior vulnerabilidades à doença causada pelo coronavírus, os idosos são colocados como grupo de risco é necessário cuidados específicos, voltadas para uma assistência de enfermagem especializada. Idosos em situações ainda maiores de risco, por apresentarem doenças preexistentes, necessitam serem acompanhados ainda mais de perto e o enfermeiro, que é o profissional responsável pelo acompanhamento em tempo integral desse paciente, precisa estar ciente em desenvolver o cuidado baseado no diagnóstico de enfermagem, prevenção da doença e promoção da saúde, a ponto de diminuir os riscos desse paciente e evitar o agravamento do covid-19 (Marins et al., 2020; Tavares et al., 2020).

O covid-19 pode desenvolver sinais e sintomas inesperados por se tratar de uma doença ainda desconhecida, em razão disso, o enfermeiro ao atender um paciente portador do covid-19 deve se preparar para encontrar inúmeras apresentações e desenvolvimento da doença, pois ainda é uma doença nova que atualmente é estudada. O idoso que apresenta uma saúde 
vulnerável pode ser acometido pela doença, na qual requer cuidados específicos da equipe de saúde, principalmente da enfermagem. Pacientes acima de 80 anos de idade, requer um atendimento mais diferenciado e com maiores cuidados, principalmente quando apresentam comorbidades. Em virtude dos grandes números de pacientes idosos em situações graves da doença, observou-se que o sistema de saúde necessita de uma reorganização estrutural, pois tiveram grande número de internação de paciente contaminados por covid-19. Além disso, há necessidade de aprimoramento do pessoal de enfermagem em relação ao cuidado específico ao idoso (Fhon et al., 2020 \& Moraes et al., 2020).

Ainda nesse contexto, reconhece-se que os idosos institucionalizados possuem um alto risco de morte por covid-19, pois são um grupo que frequentemente possui comorbidades crônicas e dificuldades para atividades da vida diária, além de contato com cuidadores, profissionais, visitantes e convivem em aglomerados. A família e a sociedade podem servir de apoio aos idosos, na qual deve orientar as ações de lavagem das mãos, uso de álcool em gel, distanciamento físico, etiqueta respiratória, cuidados ambientais e emocionais. Destacasse nessa pandemia, a essencialidade de controlar a fonte de infecção, interromper a rota de transmissão e proteger as pessoas suscetíveis (Hammerschimdt \& Santana, 2020).

Para fundamentar as ações do enfermeiro no enfrentamento ao idoso diagnosticado com covid-19, foram considerados conceitos primordiais como a pessoa, ambiente, saúde e enfermagem. Ao olhar o indivíduo sob esses aspectos, o enfermeiro pode promover medidas interventivas que atendam às necessidades deste paciente, assim como. Um planejamento realizado com toda a equipe multiprofissional pode contribuir para redução dos agravos e estimular melhorias nos fatores biopsicossociais, com melhora na saúde dos idosos contaminados pela covid-19 (Pereira et al., 2020 \& Marins et al., 2020).

\section{Cuidados de enfermagem aos idosos acometidos pelo covid-19}

A covid-19 é uma doença respiratória aguda, o qual o tratamento clínico e adequado ainda não é totalmente conhecido, porém sabe-se que o vírus possui alta transmissibilidade. Os sinais e sintomas são semelhantes a outras infecções respiratórias, com: febre; coriza; tosse, geralmente seca, e cansaço. Alguns indivíduos podem desenvolver a forma mais grave da doença, com sintomas de dispneia e/ ou sinais de sangramento pulmonar, linfopenia grave e insuficiência renal. Diante da gravidade e da alta transmissibilidade foram estabelecidas medidas preventivas, como a higienização das mãos; etiqueta respiratória; limpeza de superfícies e objetos e o distanciamento social. Porém, ao se tratar dos idosos, é notório o menor conhecimento dessa faixa etária sobre quais as medidas preventivas da covid-19 que necessita da ajuda dos enfermeiros (Oliveira; Machado e Dadalto, 2020 \& Tavares et al., 2020).

Muitos idosos realizam o autocuidado, sem ajuda de familiares ou profissionais, o que os tornam mais vulneráveis e desprovidos de apoio social diante de dificuldades de saúde. Nesse contexto, as ações educativas em enfermagem são fundamentais e devem ser planejadas e inseridas no plano de cuidado do idoso. O enfermeiro deve elaborar e executar práticas educativas com o objetivo de gerar ações de prevenção e detecção precoce das infecções pelo novo coronavírus e, assim, prevenir a evolução da doença e possíveis complicações (Oliveira, Machado, Dadalto, 2020 \& Tavares et al., 2020).

Entre as medidas para minimizar a propagação do coronavírus está o distanciamento social, que pode levar ao aparecimento de problemas mentais. Neste sentido, os idosos estão propensos ao stress causado pela quarentena. Para manter a saúde mental nessa população, pode-se utilizar diferentes estratégias, entre elas a terapia com arte, que contribui para a conexão corpo e mente (AlbañiL-Delgado; Machain \& Guerrero-Castañeda, 2020).

$\mathrm{Na}$ terapia com arte existem técnicas distintas, como as plásticas, que favorece a criatividade, autoestima e o bemestar emocional. Nesse sentido, a equipe de enfermagem e saúde, deve incentivar as artes plásticas, as quais permitem o desenvolvimento das habilidades motoras finas, para evitar a diminuição da capacidade física. Pode-se também utilizar o desenho, pinturas, colagens entre outras (Albañil-Delgado; Machain \& Guerrero-Castañeda, 2020). 
Ainda sobre isso, entre os cuidados de enfermagem e as medidas para contenção da transmissão estão as instruções para uso de EPIs, cuidados de higiene, limpeza, desinfecção de superfícies, reforço de testes diagnósticos, especialmente em lares de idosos. O apoio psicológico e suporte emocional também é essencial, visto que o distanciamento social, medida necessária como proteção, causa ansiedade e medo da solidão e da morte (Faria et al., 2020).

No momento em que falamos do risco de infecção, as pessoas que vivem em ILPI possuem um risco acrescido de infecção e desfechos adversos por viverem muito próximas de outras pessoas. A fim de proteger seus residentes, os profissionais de saúde nas instituições de longa permanência de idosos (ILPI) devem tomar algumas precauções através da utilização de atividades de prevenção e controle de infecção (PCI). As atividades PCI devem incluir treinamento no contexto da covid-19 sobre higienização das mãos e etiqueta respiratória, precauções-padrão, precauções baseadas na transmissão do vírus covid-19, sessões informativas para os residentes, estimular a higienização frequente das mãos com água e sabão ou álcool em gel. Deve-se estabelecer distanciamento mínimo para reduzir a disseminação do covid-19 (Alves \& Ferreira, 2020).

Para limitar a propagação a equipe deve identificar precocemente, isolar e encaminhar para atendimento em casos de suspeita de infecção. Também é necessário avaliar o estado de saúde de todo novo residente, na qual avalia-se os sinais de doença respiratória, febre, tosse ou falta de ar. Deve-se avaliar a temperatura corporal, presença de tosse ou falta de ar dos residentes duas vezes ao dia. Se houver suspeita de contaminação por covid-19, deve-se notificar as autoridades locais, isolar os residentes que apresentarem sintomas respiratórios, colocar máscara cirúrgica no residente e nos demais que dividem o mesmo quarto, realizar o teste para covid-19 (Alves \& Ferreira, 2020).

Em casos confirmados, o tratamento deve ser realizado prioritariamente em unidade de saúde, porém se não for possível, os cuidados de enfermagem para casos confirmados são: utilização de EPIs, priorizar um quarto individual ou agrupar os residentes positivos para covid-19, sinalizar o quarto com avisos para precaução de contato e para gotículas, separar equipamentos médicos exclusivos para os casos confirmados, limpeza e desinfecção dos equipamentos a cada utilização, restringir o compartilhamento de itens pessoais e restringir a movimentação do residente contaminado (Alves e Ferreira, 2020 \& Santana et al., 2020).

Ao considerar as milhares de mortes registradas ao redor do mundo, onde a principal faixa etária atingida foram os idosos, observa-se uma preocupação com as ILPI. Portanto, é necessário um planejamento, no qual devemos levar em consideração os recursos financeiros, materiais e humanos, que deem suporte as ações assistenciais na prevenções da infecção pela covid-19. O plano de ação baseia-se em: gerenciamento da assistência; intervenções educacionais; avaliação/monitoramento periódico de todos os residentes; prevenção e controle para impedir a disseminação do vírus; limpeza e desinfecção das superfícies, dos utensílios e produtos utilizados pelos residentes; saúde e segurança profissional; comunicação com a família; cuidado com o preparo do corpo. A partir desses pontos a equipe de enfermagem consegue organizar estratégias e executá-las, de forma que o idoso venha ser atendido de maneira integral e completa (Santana et al., 2020).

Os cuidados de enfermagem para esse tipo de paciente necessitam de uma interação enfermeiro/paciente ou família/paciente 24 horas. $\mathrm{O}$ idoso acometido pelo coronavírus, que possua algum tipo de demência, possui problemas ainda maiores para o autocuidado, além da incapacidade de identificar as necessidades. Por conta disso, o cuidado de enfermagem deve estar focado, além da assistência e checagem de sinais vitais, ao apoio familiar e psicológico desse idoso, assim como no conforto, nutrição, atenção aos transtornos de comportamento e atenção ao conforto e higiene desse paciente (Camacho, Thimoteo, Souza, 2020 \& Tavares et al., 2020).

O coronavírus trouxe uma verdadeira crise para o mundo, já que a falta de um tratamento confiável e eficaz fez com a circulação das pessoas diminuíssem. O vírus e a doença ainda estão em processo de pesquisa, algo que ainda vai perdurar por ano. Dessa forma, o enfrentamento da doença se baseou em medidas preventivas de higienização, distanciamento social e 
vacinação que exigirão novos estudos e acompanhamento. Porém, o afastamento social gerou grandes prejuízos econômicos, diversos setores ficaram comprometidos, assim como a própria qualidade de vida de cada indivíduo, que passou a sofrer um acúmulo de tensões, estresse e medo (Camacho, Thimoteo e Souza, 2020; Hammerschimd e Santana 2020; Moraes et al., 2020 \& Tavares et al., 2020).

A vista disso observa-se um aumento no número de violência familiar, pois agora, as pessoas são forçadas a convivência diária. Em razão disso, o enfermeiro tem que estar atento a qualquer tipo de violência contra o idoso, que pode ser psicológica, física, sexual, patrimonial e institucional, negligência e abuso financeiro. Os idosos se encaixam no grupo dos mais vulneráveis, pois sofrem discriminação social ao envelhecimento, dispõem de poucas políticas públicas de garantia dos seus direitos e dependem de terceiros no seu dia a dia. Portanto é de extrema importância a inclusão de políticas de enfrentamento da covid-19 no país que combata a violência contra os idosos, com políticas públicas de saúde, assistência social, apoio econômico emergencial, segurança e justiça em ações de proteção de direitos, de promoção de saúde e de detecção precoce, notificação e cuidados dos casos já instalados. Assim, haverá um controle sobre esses tipos de ocorrências e será possível manter a saúde do idoso (Hammerschimdt e Santana, 2020 \& Moraes et al., 2020).

Os idosos contaminados pelo vírus da Covid-19 que apresentarem sintomas leves a moderados da Covid-19 devem ser internados nos quartos privativos com suporte de O2. Os cuidados de enfermagem para este paciente são: proporcionar o repouso absoluto, promover um sono profundo e monitorar regularmente os sinais vitais, preferencialmente por meio de sistema de monitoramento remoto para reduzir o risco de exposição do profissional de enfermagem. Controlar a febre, como o nível ideal de temperatura ambiente, roupas leves de algodão, intervenções de resfriamento externo, como esponja morna, uso de um ventilador e esponja fria, mas lembre-se de esfriar gradualmente para evitar tremores. Estimule o paciente a tomar banho regularmente com água e sabão e a manter uma boa higiene pessoal. Fornece muitos líquidos, uma dieta nutritiva e rica em proteínas com vitaminas. Algumas das especiarias como: cúrcuma, cominho, alho, T. cordifolia ou Gilo também são consideradas eficazes na recuperação de infecções virais devido aos seus efeitos antiinflamatórios e aumenta a imunidade. Pacientes com dificuldades respiratórias podem requerer a posição de Fowler, utilize oxímetro de pulso para monitorar a saturação de oxigênio e manter sempre acima de $\mathrm{SpO} 2>$ 90\%.

No entanto, pacientes em estado crítico os cuidados são monitoramento cuidadoso da permeabilidade das vias aéreas, SpO2> 90\%, sinais vitais, nível de consciência, equilíbrio ácido-básico, ECG, perfil de coagulação, funções renais e hepáticas, sinais de TVP e risco de úlceras de pressão. Manter o paciente em posição de semifowler (30-45 de elevação da extremidade da cabeça) e troque a cada duas horas para evitar úlceras de pressão. Paciente em ventilação mecânica requer o uso de circuito ventilatório separado, prevenção de Pneumonia associada ao ventilador prevenção de infecções do trato urinário relacionadas a cateter vesical de demora, sepse relacionada a cateter venoso central. Fornecer higiene oral regularmente (a cada 6 horas com clorexidina $0,12 \%$ ), cuidados com cateter central (trocar curativo a cada 72 horas com curativo transparente), cuidados diários com os olhos, tapa-olhos, cuidados com cateter urinário, cuidados com as costas, banho no leito (com descarte esponjas molhadas). Use sistema de sucção endotraqueal fechado com baixa pressão de sucção para prevenir o risco de exposição ao aerossol.

Diante da complexidade do processo de envelhecimento humano, com peculiaridades próprias, aliada à alta incidência das doenças crônicas e suas repercussões no corpo humano, evidencia-se a necessidade de atenção específica aos idosos, o que inclui ações de prevenção, tratamento e reabilitação. Para que essa prática ocorra, é necessário que desde a formação inicial invista-se em uma abordagem adequada a respeito desse assunto.

Há uma necessidade que o enfermeiro atue ativamente na elaboração de planos de contingência, definição de plano de cuidado e que capacite os demais membros da equipe de enfermagem a identificar precocemente sinais e sintomas sugestivos de infecção. A prática clínica do enfermeiro deve ser respeitosa, centrada e individualizada, com uma abordagem que tenha 
como objetivo inicial a prevenção e em indivíduos contaminados, na melhor e mais rápida recuperação. entrado na prevenção do coronavírus como.

\section{Conclusão}

A prática clínica do enfermeiro precisou se adaptar a nova realidade imposta pela pandemia do covid-19, que não permitiu um longo acompanhamento dos pacientes para a escolha do cuidado. Na maioria dos casos, principalmente no início da pandemia, o paciente idoso contaminado pelo novo coronavírus não sobrevivia por muito tempo e a equipe de saúde conseguia apenas dar algum conforto no final da vida. Com o avanço da doença e o compartilhamento de conhecimento a respeito do assunto, os profissionais que atuam na linha de frente conseguiram desenvolver técnicas e cuidados adaptados. Em relação ao idoso portador do covid-19, cabe ressaltar a importância de um atendimento rápido e eficiente, que garanta o distanciamento do idoso acometido pela doença das demais pessoas, mas que ainda sim seja um cuidado humanizado. Novos estudos científicos são necessários para aperfeiçoar a prática clínica do enfermeiro no combate ao novo coronavírus em idosos, com foco na melhora da qualidade de vida e reabilitação para aqueles recuperados.

\section{Referências}

AlbañiL-D. S.; Machain, P. A. \& Guerrero-Castañeda, R. F. (2020). Técnicas de arteterapia en casa como cuidado de enfermería para adultos mayores en contingência por covid-19. Cogitare enfermagem. 25: e73883. http://dx.doi.org/10.5380/ce.v25i0.73883.

Alves, J. C. R \& Ferreira, M. B. (2020). Covid-19: reflexão da atuação do enfermeiro no combate ao desconhecido. Enfermagem em Foco. 11(1). 74-77.

Barros, A. L. B. L et al. (2020). Contribuições da rede de pesquisa em processo de enfermagem para assistência na pandemia de covid-19. Revista Brasileira de Enfermagem. 73(2): e20200798, 2020. https://doi.org/10.1590/0034-7167-2020-0798

Camacho, A. C. L. F.; Thimoteo, R. S. \& Souza V. M. F. (2020). Tecnologia da informação ao idoso em tempos de covid-19. 9(6): 124963497. Research, Society and Development. DOI: http://dx.doi.org/10.33448/rsd-v9i6.3497

Cesário, J. M. S.; Flauzino V. H. P.; \& Mejia J. V. C. (2020). Metodologia científica: Principais tipos de pesquisas e suas caraterísticas. Revista Científica Multidisciplinar Núcleo do Conhecimento. 5(11). 23-33. 10.32749/nucleodoconhecimento.com.br/educacao/tipos-de-pesquisas.

Faria, A. C. A et al. (2020). Covid-19: articulação das políticas de saúde e sociais para promoção de cuidados seguros aos idosos. Revista Eletrônica de Enfermagem. 22(63990). 1-8. 10.5216/ree.v22.63990.

Fhon, J. R. S et al. (2020). Atendimento hospitalar ao idoso com covid-19. Revista Latino-Americano de. Enfermagem. 28 : e3396.

John Hopkins Coronavirus Resource Center. (n.d.). United States cases by county. Johns Hopkins University \& Medicine. https://coronavirus.jhu.edu/us-map

Hammerschimdt, K. S. A. \& Santana, R. F. Saúde do idoso em tempos de pandemia covid-19. Cogitare enfermagem. 25 : e72849. http://dx.doi.org/10.5380/ce.v25i0.72849.

Lipsitch, M.; Swerdlow, D. L. \& Finelli, L. (2020). Defining the Epidemiology of Covid-19 - Studies Needed. The New England Journal of Medicine. 13(382). 1194-1196.

Lloyd-sherlock P et al. (2020). Bearing the brunt of Covid-19: older people in low- and middle-income countries. BMJ, 1(368). 1-9.

Marins, A. M. F et al. (2020). A saúde da pessoa idosa no contexto da pandemia do coronavírus: considerações para a enfermagem. Revista do Centro Oeste Mineiro, 10: e3789. 1-7. http/doi.org/10.19175/recom.v10i0.3789.

Moraes, C. L et al. (2020). Violência contra idosos durante a pandemia de Covid-19 no Brasil: contribuições para seu enfrentamento. Ciência Saúde Coletiva, 25(2). 4177-4184.

Moraes, E. N et al. (2020). Covid-19 nas instituições de longa permanência para idosos: estratégias de rastreamento laboratorial e prevenção da propagação da doença. Ciência saúde coletiva. 25(9). 3445-3458. DOI: 10.1590/1413-81232020259.20382020.

Oliveira, A. S. V.; Machado, J. C \& Dadalto, L. (2020). Cuidados paliativos e autonomia de idosos expostos à covid-19. Revista Bioética. 28(4). 595-603. $10.1590 / 1983-80422020284422$.

Oliveira, K. T et al. (2020). Principais medidas tomadas para a mudança dos processos assistenciais durante a pandemia por covid-19. Enfermagem em Foco. 11(1). 235-238. https://doi.org/10.21675/2357-707X.2020.v11.n1.ESP

Pereira, C. S et al. (2020). Medidas de enfrentamento para idosos com covid-19 a luz da teoria adaptativa de Callista Roy. Brazilian Journal of Health Review. 3(5). 13424-13435. http://dx.doi.org/10.5380/ce.v25i0.72849. 
Research, Society and Development, v. 10, n. 8, e3210816802, 2021

(CC BY 4.0) | ISSN 2525-3409 | DOI: http://dx.doi.org/10.33448/rsd-v10i8.16802

Peters, M et al. (2015). Guidance for conducting systematic scoping reviews. International International Journal of Evidence-Based Healthcare. 13(3). 141146. 10.1097/XEB.0000000000000050.

Santana, R. F. et al. (2020). Recomendações para o enfrentamento da disseminação da covid-19 em Instituições de Longa Permanência para Idosos. Revista Brasileira de Enfermagem. 73(2): e20200260. DOI: http://dx.doi.org/10.1590/0034-7167-2020-0260

Santos, J. R. L et al. (2020). Repercussões da Covid-19 para o cotidiano da pessoa idosa. REVISA. 9(1). 576-82. https://doi.org/10.36239/revisa.v9.nesp1.p576a582.

Souza, S. da S. et al. (2021). Influência da cobertura da atenção básica no enfrentamento da covid-19. Journal Health NPEPS. 6(1).1-21.

Tavares, D. M. S et al. Idosos que moram sozinhos: conhecimento e medidas preventivas frente ao novo coronavírus. Revista Latino-Americana de Enfermagem. (28): e3383. 2020. 10.1590/1518-8345.4675. 